\title{
A preliminary investigation of 4 to 11-year-old children's knowledge and understanding of stress*
}

\section{Althea Valentine, Heather Buchanan and Rebecca Knibb}

\section{Introduction}

Children's knowledge and understanding of health and illness have been widely studied. Research suggests that there are age differences in the level of children's understanding[1] although even very young children (aged 4-6) have a basic awareness of 'everyday' aspects of health and illness[2]. Children's understanding of health is multidimensional and embedded in their experiences[3]. Until recently, children's inability to explain internal processes was accounted for in terms of Piagetian theory, which suggests that children (under 7 years of age) do not have the cognitive competence to explain abstract concepts[4]. However, researchers[5] have found that children as young as five have a basic knowledge of both chicken pox and depression.

Like depression, stress is an increasingly popular abstract psychological term, which has been linked to illness[6]. Stress may be defined as a state experienced when there is a mismatch between perceived demands and perceived ability to cope[7]. There has been a wealth of research considering stress and coping in adults and young people. Children and adolescents may experience PTSD after traumatic incidents, for example, natural disasters[8] or severe illnesses[9]. However, there is a paucity of research considering how healthy children understand and cope with stress in the context of their daily life. Although research has been conducted investigating the daily stressors children face, this has tended to focus on specific life events, often using proxy ratings (e.g.[10]).

This preliminary cross-sectional, semi-structured interview study aimed to investigate children's conceptualisation of stress from 4 to 11 years of age and to examine the effects of age and experience on children's understanding, to consider whether the topic warranted further research.

\section{Methods}

\subsection{Participants}

Fifty children (18 males, 32 females) aged 4-5 ( $n=11), 6-7(n=13), 8-9(n=13)$, and 10-11 $(n=13)$ volunteered to take part from schools in the East Midlands of England, UK. There were 5 males and 8 females in each age group, apart from the youngest group, where two male participants were absent on the day of testing. Participants were from communities with predominantly white British backgrounds with English as their first language[11].

\subsection{Materials}

A semi-structured interview schedule was developed based on previous research[5]. Small adaptations were made to explore the topic of stress (Table 1 ) and the schedule piloted $(n=15)$. Children who had not heard of stress were read a gender specific story, The storybook, designed by the authors, was devised to be exactly the same for both boys and girls, except the main character in the story was made to be the same gender as the child participant. The story provided examples of stressors identified during the pilot study, e.g. falling out with friends and struggling with homework. A four-point Likert scale was used for the question, "How much can people do to stop stress?" ranging from 'nothing at all' to 'lots and lots'. 
Table 1 - Final interview questions.

1. Have you ever heard of the word stress?

If yes, continue with interview

If no, read story providing definition and examples

2. Can you tell me what you think stress means?

3. How could you tell if someone was stressed?

4. What happens when someone is stressed?

5. How did you find out about stress?

6. Do you know anybody who has been stressed?

7. Have you ever been stressed?

If yes... Can you tell me what that felt like? and Can you tell me why you think you were 'stressed'?

8. How much do you think people can do to stop stress?

9. What sort of things do you think that people could do to stop stress?

\subsection{Procedure}

The study was approved by the University Ethics Committee; informed consent was obtained from headteachers and parents. Children were told that the study would involve answering questions about stress for which there were no right or wrong answers. Children who agreed to take part were given an opportunity to play with the audiorecording equipment and informed that they could stop at any time.

Interviews (5-15 $\mathrm{min}$ ) were conducted individually in a quiet room. Prompts were used when required, without substantially deviating from the interview schedule, and interviews were audio-recorded and transcribed verbatim. The authors developed a bottom-up coding scheme, defining lower level elements before more complex elements. After reading and re-reading transcripts, these were coded for the presence or absence of identified concepts by all authors. Authors met to reach consensus for all items on which there was disagreement. Coded data was analysed using content analysis.

\section{Results}

\subsection{Children's definitions of stress}

Most children (78\%) had previously heard of the word stress; 11 younger children (aged 4-7) required the story to be read prior to defining stress. Some children across all age groups provided an appropriate definition of stress, such as, when one: 'can't do something' (behavioural), can't cope (cognitive), is worried (emotional), is tense (physical/biological), or is stressed with oneself (others). The number of children providing a definition increased with age (54\% 4-7 year olds, 100\% 8-11 year olds). As seen in Table 2, emotional factors were most frequently referred to; older children (42\% of 8-11 year olds) mentioned physical/biological components significantly more than younger children ( $8 \%$ of $4-7$ year olds), $[\chi 2(1)=7.487, p=0.006$, $p h i=0.387]$. 
Table 2 - Percentage and number of children able to define stress in each age group and elements/coping strategies that were mentioned at least once.

\begin{tabular}{|c|c|c|c|c|}
\hline & \multicolumn{4}{|l|}{ Age group } \\
\hline & $4-5(n=11)$ & $6-7(n=13)$ & $8-9(n=13)$ & $10-11(n=13)$ \\
\hline \multicolumn{5}{|l|}{ Definition of stress } \\
\hline Appropriate definition provided & $45 \%(5)$ & $62 \%(8)$ & $100 \%(13)$ & $100 \%(13)$ \\
\hline \multicolumn{5}{|l|}{ Definition included } \\
\hline Behavioural elements & $9.1 \%(1)$ & $23.1 \%(3)$ & 0 & $30.8 \%(4)$ \\
\hline Cognitive elements & 0 & $15.4 \%(2)$ & $38.5 \%(5)$ & $7.7 \%(1)$ \\
\hline Emotional elements & $27.3 \%(3)$ & $53.8 \%(7)$ & $61.5 \%(8)$ & $100 \%(13)$ \\
\hline Physical/biological elements & $9.1 \%(1)$ & $7.7 \%(1)$ & $38.5 \%(5)$ & $46.2 \%(6)$ \\
\hline Other elements & $9.1 \%(1)$ & $7.7 \%(1)$ & $15.4 \%(2)$ & $15.4 \%(2)$ \\
\hline \multicolumn{5}{|l|}{ Experience of stress } \\
\hline Children with indirect experience & $64 \%(7)$ & $85 \%(11)$ & $92 \%(12)$ & $100 \%(13)$ \\
\hline Children with direct experience & $46 \%(5)$ & $69 \%(9)$ & $77 \%(10)$ & $92 \%(12)$ \\
\hline Coping strategies & $4-5(n=9)$ & $6-7(n=8)$ & $8-9(n=12)$ & $10-11(n=13)$ \\
\hline Active coping & $33.3 \%(3)$ & $37.5 \%(3)$ & $33.3 \%(4)$ & $46.2 \%(6)$ \\
\hline Distraction & $33.3 \%(3)$ & $12.5 \%(1)$ & $8.3 \%(1)$ & $38.5 \%(5)$ \\
\hline Social support & $11.1 \%(1)$ & $37.5 \%(3)$ & $33.3 \%(4)$ & $15.4 \%(2)$ \\
\hline Emotional expression & $11.1 \%(1)$ & 0 & $41.7 \%(5)$ & $53.8 \%(7)$ \\
\hline Problem focused & $11.1 \%(1)$ & $37.5 \%(3)$ & $25 \%(3)$ & $23.1 \%(3)$ \\
\hline Emotion focused & 0 & 0 & $41.7 \%(5)$ & $23.1 \%(3)$ \\
\hline Others (e.g. take tablets, relax) & $11.1 \%(1)$ & $12.5 \%(1)$ & $58.3 \%(7)$ & $7.7 \%(1)$ \\
\hline
\end{tabular}

Definitions became more sophisticated with age. Older children tended to provide more multifaceted answers, for example, "...like when you get really bothered about something and you get really stressed and not very happy and you want to do something but you can't"[Female, 10yrs $11 \mathrm{mths}$.

\subsection{Children's experience of stress}

Most children had both indirect (86\%) and personal experience of stress (72\%), both of which increased with age[see Table 2]. Indirect experience, that is seeing someone stressed, came from a variety of sources: $57 \%$ of children had seen their mother stressed, $38 \%$ father/father figures, e.g. stepfather, $28 \%$ friends, $26 \%$ siblings, and $18 \%$ teachers.

Children reported feeling stressed for a variety of reasons. The most common of these were others as stressors, feeling left out, getting told off, and schoolwork. Older children were more likely to report schoolwork and others as stressors, whereas younger children made comments such as: "I get stressed when my toys are not being very good"[Female, 5yrs 1mth].

\subsection{Coping with stress}

Younger children were more likely than older children ( $29 \%$ of $4-7$ year olds; $4 \%$ of $8-11$ year olds) to report that there was 'nothing at all' people could do to stop stress. However, the majority of children (60\%) thought that there were 'a few things' people could do to stop stress.

Children across all age groups mentioned active coping strategies (e.g. say sorry); social support (e.g. be with others); distraction (e.g. play with toys); and problem focused strategies (e.g. confront the person stressing you) to combat stress. Only children in the upper two age groups (42\% of 8-9 year olds and $25 \%$ of $10-11$ year olds) mentioned emotion-focused coping strategies (e.g. tell self to calm down). Older children were also more likely to mention 
emotional expression as a coping strategy ( $50 \%$ of $8-11$ year olds; $8 \%$ of $4-7$ year olds). For example, "...I put my head in the pillow and start screaming. I lie on the floor and just bang my hands on the floor..." [Female, 9yrs $1 \mathrm{mth}$ ]. Some strategies, in the latter category, may be considered maladaptive, for instance: "I go to the nearest wall and hit it and punch it a few times"[Male, 11yrs $5 \mathrm{mths}]$.

\section{Discussion and conclusion}

\subsection{Discussion}

This preliminary study aimed to investigate children's conceptualisation of stress. Age differences were evident in children's understanding of stress. Older children provided more complex responses incorporating physical/biological domains; however, some of the youngest children still had a basic understanding of stress. This supports previous research exploring children's understanding of depression[5] and is in conflict with Piagetian theory, which contends that young children cannot understand abstract concepts.

Many children had experience of stress and the complexity of stressors increased with age. It is possible that children's increasing understanding was related to more extensive experience; however, due to the small sample size this was not possible to confirm.

Younger children were more likely to respond that there was nothing people can do to stop stress, similar to previous findings[1]. Educating children about adaptive coping strategies may be beneficial for dealing with more complex stressors later in childhood and would perhaps help to prevent children using the maladaptive strategies identified. However, it is appreciated that children's knowledge and understanding of stress is not necessarily how stress is experienced and coped with in reality.

Although the study was designed to be age appropriate, and incorporated interactive measures and stories, limitations should be considered. Young children's responses tended to be concise; a forced-choice method or qualitative interview may yield more fruitful responses. The study could also have been improved by asking children what they do when they are stressed. Further research may include a stress/coping questionnaire for parents.

\subsection{Conclusion}

These preliminary findings show the importance of exploring this topic further to establish the relationship between the children's knowledge, understanding, experience and coping-concerning stress.

\subsection{Practice implications}

The research could provide guidance for provider-patient communication, particularly within preventative health education helping children to cope with stress and clinically within the field of childhood PTSD.

\section{References}

[1] J.E. Bird, V.N. Podmore

Children's understanding of health and illness

Psycho Health, 4 (1990), pp. 175-185

[2] S.L. Goldman, D. Whitney-Saltiel, J. Granger, J. Rodin

Children's representations of "everyday" aspects of health and illness

J Pediatr Psychol, 16 (1991), pp. 747-766

[3] S. Normandeau, I. Kalnins, S. Jutras, D. Hanigan

A description of 5- to 12-year old children's conception of health within the context of their daily life

Psycho Health, 13 (1998), pp. 883-896

[4] J. Piaget

The child's conception of the world

Harcourt Brace, New York (1929)

[5] T. Charman, S. Chandiramani

Children's understanding of physical illnesses and psychological states

Psycho Health, 10 (1995), pp. 145-153 
[6] U. Lundberg

Stress hormones in health and illness: the roles of work and gender

Psychoneuroendocrinology, 30 (2005), pp. 1017-1021

[7] R.S. Lazarus, S. Folkman

Stress, appraisal and coping

Springer, New York (1984)

[8] M.S. Scheeringa, C.H. Zeanah

Reconsideration of harm's way: onsets and comorbidity patterns of disorders in preschool children and their caregivers following Hurricane Katrina

J Clin Child Adolesc Psychol, 37 (2008), pp. 508-518

[9] S.J. Erickson, M. Gerstle, E.Q. Montague

Repressive adaptive style and self-reported psychological functioning in adolescent cancer survivors

Child Psychiatry Hum Dev, 39 (2008), pp. 247-260

[10] G. Parker, Y. Cai, S. Tan, K. Dear, A.S. Henderson, G.T. Poh et al.

Examination stress in Singapore primary schoolchildren: how compliance by subjects can impact on study results

Acta Psychiatr Scand, 108 (2003), pp. 239-243

[11] Office for Standards in Education. Primary School Inspection Reports[online]. London: OfSTED Publications; 2004-2007. Available at: http://www.ofsted.gov.uk/[accessed December 2007].

* I confirm all patient/personal identifiers have been removed or disguised so the patient/person(s) described are not identifiable and cannot be identified through the details of the story. 